\title{
UBE2Q1 in a Human Breast Carcinoma Cell Line: Overexpression and Interaction with $\mathbf{p 5 3}$
}

\author{
Sayed Mohammad Shafiee ${ }^{1 \&}$, Mozhgan Rasti ${ }^{1 \&}$,Atefeh Seghatoleslam ${ }^{1}$, Tayebeh \\ Azimi $^{1}$, Ali Akbar Owji ${ }^{1,2 *}$
}

\begin{abstract}
The p53 tumor suppressor protein is a principal mediator of growth arrest, senescence, and apoptosis in response to a broad array of cellular damage. p53 is a substrate for the ubiquitin-proteasome system, however, the ubiquitin-conjugating enzymes (E2s) involved in p53 ubiquitination have not been well studied. UBE2Q1 is a novel E2 ubiquitin conjugating enzyme gene. Here, we investigated the effect of UBE2Q1 overexpression on the level of p53 in the MDA-MB-468 breast cancer cell line as well as the interaction between UBE2Q1 and p53. By using a lipofection method, the p53 mutated breast cancer cell line, MDA-MB-468, was transfected with the vector pCMV6-AN-GFP, containing UBE2Q1 ORF. Western blot analysis was employed to verify the overexpression of UBE2Q1 in MDA-MB-468 cells and to evaluate the expression level of p53 before and after cell transfection. Immunoprecipitation and GST pull-down protocols were used to investigate the binding of UBE2Q1 to p53. We established MDA-MB-468 cells that transiently expressed a GFP fusion proteins containing UBE2Q1 (GFP-UBE2Q1). Western blot analysis revealed that levels of p53 were markedly lower in UBE2Q1 transfected MDA-MB-468 cells as compared with control MDA-MB-468 cells. Both in vivo and in vitro data showed that UBE2Q1 co-precipitated with p53 protein. Our data for the first time showed that overexpression of UBE2Q1can lead to the repression of p53 in MDA-MB-468 cells. This repression of p53 may be due to its UBE2Q1 mediated ubiquitination and subsequent proteasome degradation, a process that may involve direct interaction of UBE2Q1with p53.
\end{abstract}

Keywords: UPS - UBE2Q1 - breast cancer - p53

Asian Pac J Cancer Prev, 16 (9), 3723-3727

\section{Introduction}

The ubiquitin-proteasome system (UPS) serves to identify and degrade unwanted intracellular proteins. This system consists of ubiquitin, three ubiquitination enzymes, and the proteasome that degrades the ubiquitintagged target proteins (Mani and Gelmann, 2005). The ubiquitination enzymes, ubiquitin activating enzymes (E1), ubiquitin conjugating enzymes (E2) and ubiquitin ligases (E3), work in concert to link a ubiquitin chain to a specific target protein. Initially ubiquitin is activated by an E1 in an ATP-dependent manner. The E1 transfers the activated ubiquitin to E2s. Ubiquitin -loaded E2s selectively interact with E3s that recruit and bind specific substrates (Dahlmann, 2007). In a few cases, however, ubiquitination may proceed without the direct involvement of E3s (Hoeller et al., 2007). As such, UPS acts as a regulator of a variety of protein substrates that play important roles in normal cellular functions and in processes of carcinogenesis including, proliferation, apoptosis, angiogenesis and motility/metastasis (Goldstein et al., 1975). Alterations in ubiquitination has been shown to be associated with destabilization of tumor suppressors (Ohta and Fukuda, 2004; Lee and Gu, 2010) and over expression of oncogenes (Wei et al., 2005; Muller and Eilers, 2008).

Contribution of the components of the UPS, specifically E2s to tumorigenesis has begun to be verified. E2 proteins like UbcH10 (also known as UBE2C) and UBE2S (also named E2-EPF) have shown to be closely linked to the cell cycle progression and hence tumorigenesis. These E2 proteins normally work with the E3 ligase APC /C (Anaphase Promoting Complex/ Cyclosome) in the regulation of cell cycle (Bremm and Komander, 2011; Tang et al., 2014).

Breast cancer is the most common malignancy in women in both developed and developing countries (Voutsadakis, 2013; Karami et al., 2014; Mermer and Turk, 2014). The importance of ubiquitination in breast cancer is highlighted by the fact that several key proteins in breast cancer such as estrogen receptor- $\alpha(\mathrm{ER} \alpha)$, progesterone receptor $(\mathrm{PR})$ and transcription factor NF- $x \mathrm{~B}$ are targets for modifications by ubiquitin and ubiquitinlike proteins. It is also reported that activation of the 
UPS through protein kinase $\mathrm{C}$ delta (PKCD) has a role in carcinogenesis of MCF-7 breast cancer cells (Zhu et al., 2013). Moreover, the protein product of BRCA1, a gene that is mutated in hereditary breast cancer syndromes is an E3 ubiquitin ligase (Voutsadakis, 2013). The implications of a number of E2 enzymes such as UBE2C and UBE2S in breast carcinoma (Bremm and Komander, 2011) and UBE2L3 (UbcH7) in breast cancer cell proliferation has been reported (Waite and Eng, 2003). UBE2B (hHR6B or Rad6B) is another E2 enzyme that is overexpressed in breast cancer (Gerard et al., 2012). This enzyme forms a complex with Mdm2, a RING finger E3 ubiquitin ligase that mediates the degradation of p53 tumor suppressor (Chen et al., 2012). The p53 is a key mediator of cell response to a broad range of stresses. This mediation is done mainly by inducing or repressing a number of genes involved in cell cycle arrest, senescence, apoptosis, DNA repair, and angiogenesis. As such, mutations of p53 are observed in various cancers including breast carcinoma (Lacroix et al., 2006). Being a substrate for the UPS, p53 is maintained at low levels by continual proteolytic degradation in normal cells (Yang et al., 2004). In cancer cells, p53 function can be altered by mechanisms that include mutations in the p53 gene and alteration of the p53 regulator proteins (Lacroix et al., 2006). p53 mutations are reported in the familial Li-Fraumeni syndrome which is characterized by a high incidence of multiple early-onset cancers including breast tumors. Other hereditary breast cancers may be due to mutations in genes coding for p53 regulator proteins (Lacroix et al., 2006). A significant proportion of these cancers have been associated with mutations of BRCA1 (Hohenstein and Giles, 2003). p53 mediates its own down-regulation by inducing the expression of Mdm2 that is an E3 ubiquitin-protein ligase. Recently, it is reported that Mdm2 cooperates with different E2 enzymes to regulate the stability and function of p53 (Moll and Petrenko, 2003). E2 enzymes like UBE2D2 (UbcH5b) and UBE2D3 have also shown to mediate the ubiquitination and down-regulation of p53 (Saville et al., 2004).

Recently we have reported that the novel human gene, UBE2Q1, is a putative member of E2 family of enzymes and is differentially expressed in breast tumors (Seghatoleslam et al., 2012b), colorectal cancer (Shafiee et al., 2013) and pediatric acute lymphoblastic leukemia (Seghatoleslam et al., 2014). UBE2Q1 is very similar to another human protein, UBE2Q2 that has already been shown to be overexpressed in malignancies such as head and neck squamous cell carcinoma (Seghatoleslam et al., 2006), breast cancer (Nikseresht et al., 2010), acute lymphoblastic leukemia (Seghatoleslam et al., 2012a) and colorectal primary tumors (Shafiee et al., 2014). Overexpression of UBE2Q2 can negatively affect cell proliferation and anchorage-independent cell growth (Maeda et al., 2009; Seghatoleslam and Zambrano, 2009). A ubiquitin conjugating enzyme E2 domain is predicted at the C-terminal of both UBE2Q1 and UBE2Q2 proteins (Seghatoleslam et al., 2006; Seghatoleslam et al., 2012b).

In the present study we investigated the effect of UBE2Q1 overexpression on the level of p53 in MDAMB-468 line of breast cancer cells and also checked the interaction of UBE2Q1 protein with native and mutant $\mathrm{p} 53$ in MCF7 and MDA-MB-468 cells respectively.

\section{Materials and Methods}

\section{Cell culture}

Breast cancer cell lines MCF7 (p53-positive) and MDA-MB-468 (p53-mutant) were obtained from national cell bank of Iran (Pasture Institute, Iran). Cell lines were cultured in $10 \%$ fetal calf serum (Cinagen, Iran) containing media (Biosera, UK) of RPMI 1640 and maintained under an atmosphere of $5 \% \mathrm{CO} 2$ in a humid incubator at $37^{\circ} \mathrm{C}$.

\section{Cell transfection}

Transfections of MDA-MB-468 cells with UBE2Q1 cDNA were performed by the lipofection method using Lipofectamine ${ }^{\circledR} 2000$ transfection reagent (Invitrogen, USA) according to the manufacturer's procedure. Human UBE2Q1 ORF cDNA clone (NM_017582.6) in pCMV6AN-GFP vector was from Origene (USA). The control transfection was performed by pCMV6-AN-GFP vector without UBE2Q1 ORF. Overexpression of UBE2Q1 by these cells was verified by Western blot analysis using anti UBE2Q1 antibody as discussed below.

\section{Protein extraction and Western blot analysis}

To extract protein from cultured cells, after detaching the cells from the flasks using cell scraper, they were lysed in extraction buffer containing $150 \mathrm{mM}$ sodium chloride, $1.0 \% \mathrm{NP}-40(\mathrm{~V} / \mathrm{V}), 50 \mathrm{mM}$ Tris, $\mathrm{pH} 8.0$ and protease inhibitor cocktail (Roche, Germany). Lysates were sonicated at $4^{\circ} \mathrm{C}$ for $30 \mathrm{~s}$ and then maintained in constant agitation for 30 minutes at $4^{\circ} \mathrm{C}$ and finally cleared by centrifugation. Protein concentration was measured using Bradford reagent. Electrophoresis (MiniPROTEAN Tetra Cells BioRad, USA) of $30 \mu \mathrm{g}$ protein samples were performed on $12.5 \%$ discontinuous sodium dodecyl sulfate-polyacrylamide gel electrophoresis (SDS- PAGE) and the proteins were transferred onto nitrocellulose membrane (PROTRAN Nitrocellulose transfer Membrane, Whatman, USA) at $25 \mathrm{~V}$ for $18 \mathrm{~h}$ in cold room (Mini Trans-Blot Cell, BioRad, USA). The membranes were blocked for $1 \mathrm{~h}$ in $5 \%$ dried fat free milk, at room temperature and incubated with specific primary antibodies diluted in blocking solution (1:2,000 for anti UBE2Q1 antibody and 1:500 for anti p53 antibody) at $4{ }^{\circ} \mathrm{C}$ overnight. The anti UBE2Q1 antibody was a polyclonal rabbit antiserum generated against the peptide ATDRLMKELRDIYRSQSF corresponding to amino acid sequences 252-269 of UBE2Q1 (Seghatoleslam et al., 2012b). Anti-p53 antibody was also generated in our recombinant protein laboratory (Rasti et al., 2012). Blots were washed three times in PBS-Tween (PBS-T) and incubated with specific secondary antibodies coupled to HRP (HRP-conjugated goat anti-rabbit IgG, Abcam, USA) $(1: 2,500)$ at a concentration of $1 \mu \mathrm{g} / \mathrm{ml}$ in $2 \%$ (W/V) BSA in PBS-T. All samples were also blotted for $\beta$-actin (1:1000) to normalize the amounts of protein. A chemiluminescent substrate (Chemiluminescent Kit, BioRad, USA) were used for detecting the bands on 
membranes. Light emission was captured by exposing the membrane to X-ray films.

\section{Immunoprecipitation and GST pull-downs}

MCF7 and MDA-MB-468 breast cancer cell lines were lysed in a buffer containing $10 \mathrm{mM}$ Tris- $\mathrm{HCl} \mathrm{pH} \mathrm{7.4,0.825}$ $\mathrm{M} \mathrm{NaCl}$ and $1 \%(\mathrm{v} / \mathrm{v}) \mathrm{NP}-40$. Cell lysates were sonicated and cleared by centrifugation. Five milligrams of the protein lysate was used for immunoprecipitation and GST pull-down studies. Immunocomplexes were precipitated using $5 \mu \mathrm{l}$ of polyclonal rabbit antiserum against either p53 (Rasti et al., 2012) or UBE2Q1 (Seghatoleslam et al., $2012 \mathrm{~b}$ ). After 2 hours of mixing at $4^{\circ} \mathrm{C}$ on a rotator, $30 \mu \mathrm{l}$ of packed protein $\mathrm{G}$ agarose beads (Sigma, UK) were added to the protein-antibody complex and mixed for a further 1 hour. Immunocomplexes bound to the beads were then spun and washed, prior to re-suspension in the SDS sample buffer for SDS-PAGE analysis. Western blotting was performed by using antisera against p53 and UBE2Q1. For GST pull-downs, $50 \mu \mathrm{g}$ of GST-p53 which were made in our lab (Rasti et al., 2012) was mixed with each cell lysates for 2 hours at $4^{\circ} \mathrm{C}$. GST fusion protein complexes were eluted from glutathione-agarose columns by 25 $\mathrm{mM}$ reduced glutathione in $50 \mathrm{mM}$ Tris- $\mathrm{HCl}$ solution $\mathrm{pH}$ 8.0. The samples were resolved by SDS-PAGE and then subjected to Western blot analysis with UBE2Q1 antibody.

\section{Results}

Effects of UBE2Q1 overexpression on the cellular levels of $p 53$

MDA-MB-468 cells were transfected by pCMV6-ANGFP vector, containing UBE2Q1 coding sequence and also by the empty pCMV6-AN-GFP vector as a control. Western blot analysis of lysates from GFP-UBE2Q1 transfected MDA-MB-468 cells revealed an intense band of UBE2Q1 immunoreactivity at about $70 \mathrm{KD}$ that is close to the molecular weight $(23 \mathrm{KD}+46.127 \mathrm{KD})$ expected for GFP tagged UBE2Q1 (Figure 1A). Therefore we established MDA-MB-468 cells that transiently expressed a GFP fusion protein containing UBE2Q1 (GFPUBE2Q1). Western blot analysis revealed that levels of p53 were markedly lower in UBE2Q1 transfected MDAMB-468 cells as compared with control MDA-MB-468 cells (Figure 1B).

\section{Interaction between $p 53$ and $U B E 2 Q 1$}

We studied the binding of UBE2Q1 to wild type (WT) p53 and the mutant p53 in MCF7 and MDA-MB-468 cells respectively. To verify these interactions, we carried out in vitro and in vivo binding studies. In vivo binding assays were performed by preparing lysates from MDAMB-468 and MCF7 cells. Lysates from these cells were immunoprecipitated by anti-p53 or with anti-UBE2Q1 antibodies and subjected to Western blotting afterwards. The blots were probed with the reciprocal antisera. Results showed that both WT and mutant p53 are able to coimmunoprecipitate with UBE2Q1. This interaction was not detected when rabbit immunoglobulin $\mathrm{G}$ was used as a control for non-specific immunoprecipitation (Figure $2 \mathrm{~A})$. For in vitro binding assays GST-p53 was expressed
A 12 3
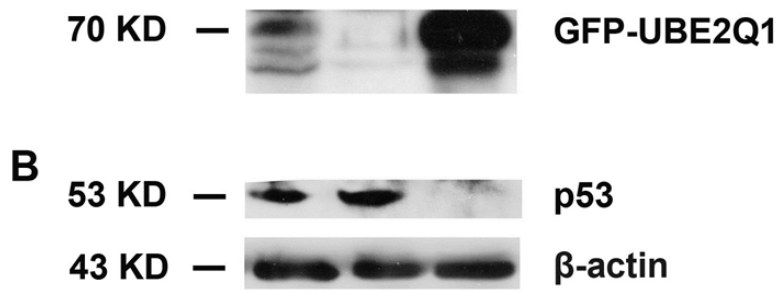

Figure 1. Expression of GFP-UBE2Q1 and p53 in MDA-MB-468 Cells. Lysates from nontransfected (lane 1), empty vector-transfected (lane2) and GFP-UBE2Q1 transfected MDA-MB-468 cells (lane 3 ) were Western blotted and probed with antibodies against UBE2Q1 (A) and p53 (B). Anti- $\beta$-actin antibody was used to confirm equal loading

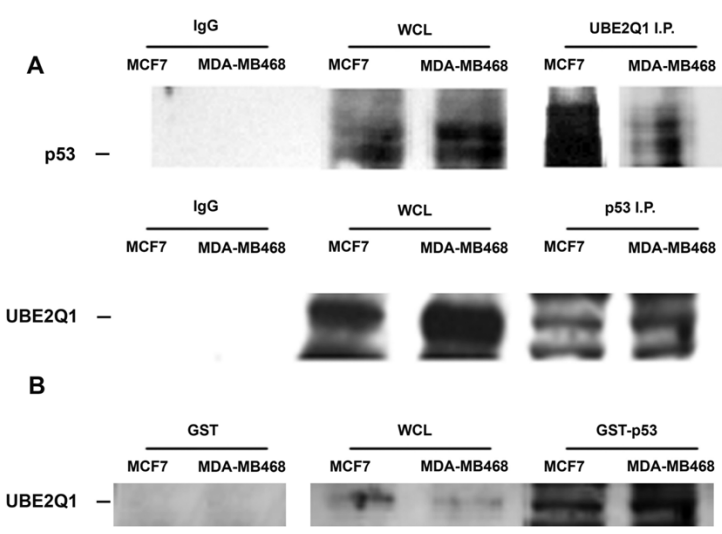

Figure 2.Western Blot Analysis of the Interaction of UBE2Q1 with WT and/or Mutant p53 Respectively in MCF7 and MDA-MB-468 Cells. A- Coimmunoprecipitation of UBE2Q1 and p53. UBE2Q1 complexes were immunoprecipitated from MCF7 and MDA-MB-468 cellular lysates by anti-UBE2Q1 (upper panel) and anti-p53 (lower panel) antibodies. The same samples incubated with rabbit immunoglobulin $\mathrm{G}(\mathrm{IgG})$ were included as a control to verify the specificity of the co-immunoprecipitation. The coexistence of p53 and UBE2Q1 in the immunocomplex was revealed by Western blot analysis using antibodies against p53 (upper panel) and UBE2Q1 (lower panel). B- Results of the GST pull down showed the interaction between GST-p53 and UBE2Q1. GST-p53 was used to pull-down protein partners in extracts from MCF7 and MDA-MB-468 cells. Anti-UBE2Q1 antibody was used to detect UBE2Q1 in complex with GST-p53. WCL: whole-cell lysate; I.P.: immunoprecipitant

in E. coli and purified with glutathione-agarose beads (Rasti et al., 2012). A pull-down assay was performed by incubating the GST-p53 with lysates from each of the cancer cell lines. Protein complexes were subjected to Western blotting and probed by anti-UBE2Q1 antibody. As shown in Figure 2B, UBE2Q1 was able to coprecipitate with GST-p53 but not with GST.

\section{Discussion}

Results of this study imply that UBE2Q1, a novel putative E2 enzyme, interacts with the p53 tumor suppressor protein. The regulation of p53 turnover by the UPS is a key issue in the understanding of molecular basis of tumorigenesis (Yang et al., 2004). Ubiquitination 
of proteins, however, signals cellular processes other than proteolysis that also participate in the development of cancer (Ohta and Fukuda, 2004; Mani and Gelmann, 2005; Dahlmann, 2007). Many proteins implicated in malignancies including breast cancer are involved in the UPS (Ohta and Fukuda, 2004). Central in the UPS are E2s that interact with several E3 ubiquitin ligases (Mani and Gelmann, 2005). Mdm2 is an E3 enzyme which has a central role in degradation of $\mathrm{p} 53$ as a tumor suppressor (Devine and Dai, 2012). Thus, exploration of E2 partners of Mdm2 as well as E2s that directly interact with p53 isimportant, particularly to understand the roles of E2s in p53 turnover and the process of carcinogenesis. Here, we sought to study the possible interaction of UBE2Q1with wild type (WT) p53 and the mutant p53 in MCF7 and MDA-MB-468 cells respectively.

Data of our study clearly shows that overexpression of the novel E2 enzyme, UBE2Q1, leads to the repression of p53 in MDA-MB-468 cells. This repression of p53 may be due to its UBE2Q1 mediated ubiquitination and subsequent proteasome degradation, a process that may involve direct interaction of UBE2Q1with p53.

Our in vivo co-immunoprecipitation results showed that both WT and mutant p53 expressed respectively in MCF7 and MDA-MB-468 cells co-immunoprecipitated with UBE2Q1.

This finding suggests that UBE2Q1 and p53 can associate into a complex. Results of subsequent GST pulldown experiments implied that co-precipitation of the two proteins may be a result of a direct interaction between them. The fact that p53 gene in MDA-MB-468 cells is mutated at codon 273 (Lacroix et al., 2006) implies that this mutation in p53 does not affect its binding capacity to UBE2Q1. This may be because this mutation affects the DNA binding region (residues 103-292), i.e. the central core region of the protein (Lacroix et al., 2006), whereas the C-terminal lysine residues are suggested to be the main sites of the ubiquitin ligation, that target p53 for proteasome-mediated degradation (Rodriguez et al., 2000). Our previous data also showed that upregulation of UBE2Q1 at the levels of both protein and mRNA is a frequent phenomenon in breast cancer (Seghatoleslam et al., 2012b). Thus, it would be plausible that up-regulation of UBE2Q1 confers a benefit on cancer cells by decreasing the level of $\mathrm{p} 53$. This finding is in agreement with the reports in which UBE2Q1 silencing by small interfering RNA caused a significant increase in the levels of p53 and p21 in HepG2 and BEL-7404 cells (Chang et al., 2014) and also the overexpression of UBE2Q1 resulted in decreased cellular level of p53 in PC12 neuronal cells (Wan et al., 2014). It is well established that Mdm 2 cooperates with different E2 enzymes for the regulation of p53 stability and function (Lai et al., 2002; Brooks and Gu, 2003). The role of Mdm2, however, in the repression of $\mathrm{p} 53$ by UBE2Q1 needs to be confirmed.

To our knowledge this is the first report of binding of UBE2Q1 to $\mathrm{p} 53$ protein in vivo and in vitro. $\mathrm{p} 53$ might be one of the UBE2Q1 substrates for ubiquitination.
This study has been supported by Grant Number 926729 from Vice-chancellor for Research Affairs of Shiraz University of Medical Sciences, Shiraz, Iran.

\section{References}

Bremm A, Komander D (2011). Emerging roles for Lys 11-linked polyubiquitin in cellular regulation. Trends Biochem Sci, 36, 355-63.

Brooks CL, Gu W (2003). Ubiquitination, phosphorylation and acetylation: the molecular basis for p53 regulation. Curr Opin Cell Biol, 15, 164-71.

Chang R, Wei L, Lu Y, et al (2015). Upregulated expression of ubiquitin-conjugating enzyme E2Q1 (UBE2Q1) is associated with enhanced cell proliferation and poor prognosis in human hapatocellular carcinoma. J Mol Histol, 46, 45-56.

Chen S, Wang DL, Liu Y, et al (2012). RAD6 regulates the dosage of p53 by a combination of transcriptional and posttranscriptional mechanisms. Mol Cell Biol, 32, 576-87.

Dahlmann B (2007). Role of proteasomes in disease. BMC Biochem, 8,3 .

Devine T, Dai MS (2013). Targeting the ubiquitin-mediated proteasome degradation of $\mathrm{p} 53$ for cancer therapy. Curr Pharm Des 19, 3248-62.

Gerard B, Sanders MA, Visscher DW, et al (2012). Lysine 394 is a novel Rad6B-induced ubiquitination site on beta-catenin. Biochim Biophys Acta, 1823, 1686-96.

Goldstein G, Scheid M, Hammerling U, et al (1975). Isolation of a polypeptide that has lymphocyte-differentiating properties and is probably represented universally in living cells. Proc Natl Acad Sci U S A, 72, 11-5.

Hoeller D, Hecker CM, Wagner S, et al (2007). E3-independent monoubiquitination of ubiquitin-binding proteins. Mol Cell, 26, 891-8

Hohenstein P, Giles RH (2003). BRCA1: a scaffold for p53 response? Trends Genet, 19, 489-94.

Karami K, Cheraghi M, Amori N, et al (2014). Common cancers in Khuzestan province, south west of Iran, during 2005-2011. Asian Pac J Cancer Prev, 15, 9475-8.

Lacroix M, Toillon RA, Leclercq G (2006). p53 and breast cancer, an update. Endocr Relat Cancer, 13, 293-325.

Lai Z, Yang T, Kim YB, et al (2002). Differentiation of Hdm2mediated p53 ubiquitination and $\mathrm{Hdm} 2$ autoubiquitination activity by small molecular weight inhibitors. Proc Natl Acad Sci USA, 99, 14734-9.

Lee JT, Gu W (2010). The multiple levels of regulation by p53 ubiquitination. Cell Death Differ, 17, 86-92.

Maeda H, Miyajima N, Kano S, et al (2009). Ubiquitinconjugating enzyme UBE2Q2 suppresses cell proliferation and is down-regulated in recurrent head and neck cancer. Mol Cancer Res, 7, 1553-62.

Mani A, Gelmann EP(2005). The ubiquitin-proteasome pathway and its role in cancer. J Clin Oncol, 23, 4776-89.

Moll UM, Petrenko O (2003). The MDM2-p53 interaction. Mol Cancer Res, 1, 1001-8.

Muller J, Eilers M (2008). Ubiquitination of myc: proteasomal degradation and beyond. Ernst Schering Found Symp Proc, 2008, 99-113.

Nikseresht M, Seghatoleslam A, Monabati A, et al (2010). Overexpression of the novel human gene, UBE2Q2, in breast cancer. Cancer Genet Cytogenet, 197, 101-6.

Ohta T, Fukuda M (2004). Ubiquitin and breast cancer. Oncogene, 23, 2079-88.

Rasti M, Arabsolghar R, Khatooni Z, et al (2012). p53 Binds to estrogen receptor 1 promoter in human breast cancer cells.

\section{Acknowledgements}


Pathol Oncol Res, 18, 169-75.

Rodriguez MS, Desterro JM, Lain S, et al (2000). Multiple C-terminal lysine residues target p53 for ubiquitinproteasome-mediated degradation. Mol Cell Biol, 20, 8458-67.

Saville MK, Sparks A, Xirodimas DP, et al (2004). Regulation of p53 by the ubiquitin-conjugating enzymes $\mathrm{UbcH} 5 \mathrm{~B} / \mathrm{C}$ in vivo. J Biol Chem, 279, 42169-81.

Seghatoleslam A, Bozorg-Ghalati F, Monabati A, et al (2014). UBE2Q1, as a down regulated gene in pediatric acute lymphoblastic leukemia. Int J Mol Cell Med, 3, 95-101.

Seghatoleslam A, Monabati A, Bozorg-Ghalati F, et al (2012a). Expression of UBE2Q2, a putative member of the ubiquitinconjugating enzyme family in pediatric acute lymphoblastic leukemia. Arch Iran Med, 15, 352-5.

Seghatoleslam A, Nikseresht M, Shafiee SM, et al (2012b). Expression of the novel human gene, UBE2Q1, in breast tumors. Mol Biol Rep, 39, 5135-41.

Seghatoleslam A, Zambrano A (2009). Effects of over-expression of LOC92912 gene on cell cycle progression. Iran J Med Sci, 34, 227-84.

Seghatoleslam A, Zambrano A, Millon R, et al (2006). Analysis of a novel human gene, LOC92912, over-expressed in hypopharyngeal tumours. Biochem Biophys Res Commun, 339, 422-9.

Shafiee SM, Seghatoleslam A, Nikseresht M, et al (2013). UBE2Q1 expression in human colorectal tumors and cell lines. Mol Biol Rep, 40, 7045-51.

Shafiee SM, Seghatoleslam A, Nikseresht M, et al (2014). Expression status of UBE2Q2 in colorectal primary tumors and cell Lines. Iran J Med Sci, 39, 196-202.

Tang XK, Wang KJ, Tang YK, et al (2014). Effects of ubiquitinconjugating enzyme $2 \mathrm{C}$ on invasion, proliferation and cell cycling of lung cancer cells. Asian Pac J Cancer Prev, 15, 3005-9.

Voutsadakis IA (2013). Ubiquitin- and ubiquitin-like proteinsconjugating enzymes (E2s) in breast cancer. Mol Biol Rep, 40, 2019-34.

Waite KA, Eng C (2003). BMP2 exposure results in decreased PTEN protein degradation and increased PTEN levels. Hum Mol Genet, 12, 679-84.

Wan C, Chen J, Hu B, et al (2014). Downregulation of UBE2Q1 is associated with neuronal apoptosis in rat brain cortex following traumatic brain injury. J Neurosci Res, 92, 1-12.

Wei W, Jin J, Schlisio S, et al (2005). The v-Jun point mutation allows c-Jun to escape GSK3-dependent recognition and destruction by the Fbw7 ubiquitin ligase. Cancer Cell, 8 , 25-33.

Yang Y,Li CC, Weissman AM (2004). Regulating the p53 system through ubiquitination. Oncogene, 23, 2096-106.

Zhu S, Yao F, Li WH, et al (2013). PKC-dependent activation of the ubiquitin proteasome system is responsible for high glucose-induced human breast cancer MCF-7 cell proliferation, migration and invasion. Asian Pac J Cancer Prev, 14, 5687-92. 